\title{
Planejamento do transporte de empregados por uma frota de ônibus fretada por meio de um modelo matemático baseado no Open Vehicle Routing Problem (OVRP)
}

\author{
Rayane Florentina Scárdua ${ }^{1}$, Rodrigo de Alvarenga Rosa ${ }^{2}$, Jodelson Aguilar Sabino ${ }^{3}$ e Lirielly Ruela Vitorugo ${ }^{4}$
}

\begin{abstract}
Resumo: O cenário da mobilidade urbana atual causa à sociedade vários problemas de transporte e qualidade de vida, tais como: altos níveis de congestionamento, lotações em transportes públicos, insuficiência de estacionamento e poluição. O transporte de passageiros por fretamento surge como alternativa para a solução dos problemas de mobilidade podendo colaborar para diminuir os congestionamentos e a emissão de poluentes. Algumas empresas oferecem como benefício a seus empregados o transporte de ida e volta do trabalho por ônibus fretados. Assim, é proposto nesse artigo uma adaptação de um modelo matemático baseado no Open Vehicle Routing Problem (OVRP) para o planejamento do transporte de empregados por meio de uma frota de ônibus fretada visando à redução do custo total gasto pela empresa. O modelo foi aplicado a uma empresa localizada em Vitória-ES e os resultados obtidos pelo modelo indicaram uma redução no custo de transporte quando comparado ao atualmente pago pela empresa.

Palavras-chave: Open Vehicle Routing Problem (OVRP), transporte Fretado de passageiros, logística urbana.
\end{abstract}

\begin{abstract}
Several transportation problems like traffic jam, crowded public transportation, parking shortage and pollution is caused by the actual scenario of urban mobility. The transport of passengers by charter is an alternative to improve the quality of urban mobility avoiding traffic jam and reducing pollution. Several companies offer as a benefit to their employees this type of transport to carry them to the company from their home and vice versa. Thus, it is proposed in this paper an adaptation of a mathematical model based on Open Vehicle Routing Problem (OVRP) for planning the transport of employees by a chartered bus fleet in order to reduce the total cost spent by the company. The model was applied to a company located in Vitória-ES and the results obtained by the model indicated a reduction in the cost of transportation when compared to the currently paid by the company.
\end{abstract}

Keywords: Open Vehicle Routing Problem (OVRP), chartered passenger transportation, urban logistics.

\section{INTRODUÇÃO}

A qualidade da mobilidade urbana tem se degradado nos últimos anos por conta de alguns fatores, dentre eles, podem ser citados: a baixa qualidade do transporte público urbano, a falta de incentivo à utilização de transporte coletivo ou não motorizados, o aumento da frota de veículos automotores, a carência de planejamento urbano e a gestão pública pouco eficaz são alguns entraves ao progresso deste setor. Vários problemas de transporte e qualidade de vida são enfrentados diariamente pela sociedade brasileira, tais como: altos níveis de congestionamento, lotações em transportes públicos, falta de acessibilidade, insuficiência de estacionamento, demora nos deslocamentos, elevados índices de acidente de trânsito e degradação ambiental (Pinto, 2012; Vasconcellos, 2012).

Nota-se, também, que uma alternativa eficiente para se complementar o transporte público poderia ser o transporte de passageiros por fretamento. Este tipo de transporte resulta em menor consumo de espaço viário e menor emissão de poluentes, contribuindo também para a redução dos

\footnotetext{
${ }^{1}$ Rayane Florentina Scárdua, Programa de Engenharia Civil, UFES. (rayanescardua@gmail.com)

2 Rodrigo de Alvarenga Rosa, Programa de Engenharia Civil, UFES.

(rodrigoalvarengarosa@gmail.com)

3 Jodelson Aguilar Sabino, Programa de Engenharia Civil, UFES

(jodelson.sabino@gmail.com)

${ }^{4}$ Lirielly Ruela Vitorugo, Programa de Engenharia Civil, UFES

(Ivitorugo@gmail.com)
}

Manuscrito recebido em 06/03/2016 e aprovado para publicação em $25 / 08 / 2016$

Este artigo é parte de TRANSPORTES v. 24, n. 3, 2016. ISSN: 2237-1346 (online). DOI:10.4237/transportes.v24i3.1089 níveis de congestionamentos (Pinto, 2012; Vasconcellos, 2012). Algumas empresas oferecem como benefício a seus empregados o serviço de transporte de ida e volta do trabalho por veículos fretados. Esse serviço oferece vantagens tanto aos passageiros quanto às empresas uma vez que, por um lado, proporciona aos empregados segurança, comodidade e rapidez nos deslocamentos e, por outro, garante às empresas o aumento da produtividade dos trabalhadores, já que reduz o número de faltas e atrasos no serviço.

As empresas que contratam o transporte por fretamento para seus empregados, são, geralmente, responsáveis pela concepção das rotas e a sua gestão. Na maioria das empresas, a criação de rotas é feita de forma empírica, baseada na experiência de alguns empregados. Uma característica deste tipo de transporte é o aluguel de uma frota de ônibus cujo custo é calculado em função do número de veículos utilizados e da quilometragem percorrida por estes.

Dentre as várias classes do Vehicle Routing Problem (VRP), a que se mostra mais adequada a este problema é o Open Vehicle Routing Problem (OVRP), que difere do VRP pelo fato de os veículos não retornarem ao depósito (nesse caso a garagem) após atenderem o último cliente. Entre os estudos já realizados sobre o OVRP, o trabalho de Bektas e Elmastas (2007) é o que mais se assemelha ao problema de transporte de empregados por uma frota de ônibus fretada, porém, ele foi aplicado ao problema de transporte escolar. Desta forma, utilizou-se o modelo proposto por estes autores com o intuito de reduzir o custo total de transporte de empregados por meio de uma frota de ônibus fretada. $\mathrm{O}$ modelo proposto difere do modelo de Bektas e Elmastas (2007) pelo fato de introduzir limites inferiores e superiores para o número de ônibus utilizados no transporte. 


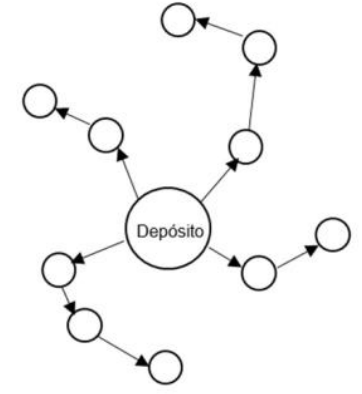

(a) Ilustração de caminho Hamiltoniano
Figura 1. Legenda

Fonte: Brandão, 2004

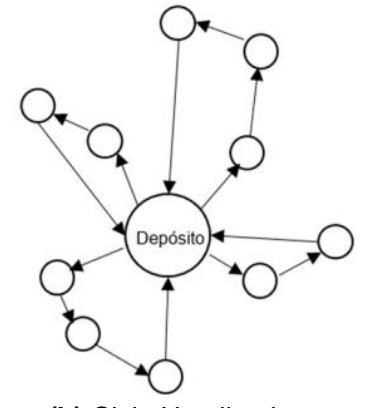

(b) Ciclo Hamiltoniano
Essa adaptação foi feita com o intuito de restringir o espaço de soluções, o que reduz substancialmente o tempo de execução do modelo.

O modelo proposto foi aplicado ao planejamento do transporte diário de empregados por uma frota de ônibus fretada contratada por uma empresa de grande porte, tendo sido possível constatar, com essa aplicação, a eficiência da solução para um caso real. O transporte é realizado nos percursos de casa para o trabalho e do trabalho para casa e engloba os turnos de trabalho operacional e administrativo.

Esse artigo está organizado em cinco seções, a começar por essa introdução. Na Seção 2 tem-se a definição do Problema de Transporte de Passageiros por Fretamento e a revisão da literatura. A Seção 3 apresenta o modelo matemático proposto. A Seção 4 descreve a metodologia, a apresentação e as análises dos resultados encontrados e, por último, a Seção 5 apresenta as principais conclusões do trabalho.

\section{PROBLEMA DE TRANSPORTE DE PASSAGEIROS POR FRETAMENTO}

As empresas geralmente adotam algumas premissas para o transporte de seus empregados como o número máximo de passageiros por ônibus e o tempo máximo de viagem. Em geral, a quilometragem contabilizada para tarifação se inicia no primeiro ponto de atendimento ao passageiro, o que desconsidera o trecho entre a garagem e o primeiro ponto. Quanto aos custos de transporte, estes são classificados em duas categorias: Custos Fixos e Custos Variáveis. No caso do fretamento, o custo fixo usualmente é o custo do aluguel do ônibus e o custo variável refere-se aos quilômetros percorridos pela frota. Considerando os transportes de ida e volta do trabalho, o problema de roteirização dos veículos por fretamento pode ser dividido em dois subproblemas: 1) Coleta de empregados no percurso de ida ao local de trabalho e 2) Distribuição de empregados no percurso de volta do local de trabalho.

$\mathrm{Na}$ literatura, o problema que mais se assemelha ao transporte de passageiros por uma frota fretada é o OVRP, que difere do VRP pelo fato de os veículos não serem obrigados a retornar ao depósito após visitarem o último cliente (Letchford et al., 2007). Essa diferença é ilustrada pela Figura 1. No OVRP, cada rota é um caminho Hamiltoniano, formando rotas abertas, Figura 1 (a), enquanto no VRP, estas definem um ciclo Hamiltoniano, caracterizando rotas fechadas, Figura 1 (b) (Brandão, 2004).

No OVRP, cada cliente é visitado exatamente uma vez por um veículo, e a atividade dos veículos é limitada por restrições de capacidade e de tempo. Na literatura, este problema possui geralmente dois objetivos: minimizar a distância total percorrida ou o tempo de viagem total dispendido pelos veículos, e o número de veículos utilizados. Traduzindo em termos de custo, isso equivale a minimizar o custo operacional dos veículos e o custo total de viagem (De Jaegere et al. 2014).

A seguir é realizada uma revisão da literatura sobre o OVRP e o transporte de empregados por fretamento. Bodin et al. (1983) propuseram a primeira abordagem para solucionar o OVRP aplicado à distribuição do correio aéreo nos EUA, onde o problema foi denominado Open Pick-Up and Delivery VRP com restrições de capacidade e janelas de tempo. Sarikilis e Powell (2000) propuseram uma heurística baseada em uma árvore geradora mínima (Minimum Spanning Tree - MST) para o OVRP.

Tarantilis e Kiranoudis (2002) abordaram um problema real de uma grande indústria grega para distribuir carnes frescas dos depósitos a seus clientes. Esse problema foi formulado como um problema do tipo Open MultiDepot Vehicle Routing Problem (OMDVRP). Para resolver o problema, os autores propuseram um novo algoritmo ListBased Threshold Accepting (LBTA). Brandão (2004) desenvolveu uma meta-heurística Tabu Search (TS) para resolver o OVRP. Fu et al. (2005) aplicaram o OVRP para o planejamento de trens que percorrem o Eurotúnel com restrições de capacidade e janela de tempo e para o planejamento de rotas de ônibus escolares em que os alunos são coletados de manhã em vários locais e entregues à escola e, à tarde, as rotas são inversas para levá-los até suas casas. Letchford et al. (2007) apresentaram um método Branch-and-Cut para o OVRP e os resultados mostraram que para instâncias de pequena e média escala, o OVRP é tão passível de solução exata quanto o VRP. Li e Tian (2006) aplicaram um método híbrido composto por Ant Colony System (ACS) e Busca Local para solucionar o OVRP. Li et al. (2007) compararam os resultados de onze algoritmos encontrados na literatura para o OVRP e constataram que os procedimentos baseados em Adaptive Large Neighborhood Search, Record-to-Record Travel e TS tiveram bom desempenho. Zachariadis e Kiranoudis (2010) apresentaram uma meta-heurística de Busca Local que avalia um vasto conjunto de soluções visinhas para solucionar o OVRP. Para explorar este conjunto de soluções visinhas com um esforço computacional razoável, os movimentos da busca local são estaticamente codificados em entidades SMD (Static Move Descriptor).

A ideia central do SMD é que quando move-se de uma solução para uma outra solução, apenas uma parte limitada da solução é modificada. Portanto, para examinar 
uma próxima visinhança de solução, apenas os movimentos que estão associados com a parte afetada da solução têm que ser re-avaliados.

Salari et al. (2010) apresentaram um procedimento de melhoria para o OVRP baseado em técnicas de Programação Linear Inteira, utilizaram de instâncias presentes na literatura, em que para várias destas instâncias o algoritmo proposto encontrou soluções melhores que as já encontradas anteriormente por outros métodos. Yu et al. (2011) solucionaram o OVRP por meio da aplicação de um método híbrido baseado em Algoritmo Genético e TS. Bektas e Elmastas (2007) apresentaram um um modelo matemático para resolver o School Bus Routing Problem (SBRP). O problema diz respeito ao transporte de estudantes de uma escola primária em Arkara, Turquia, e foi formulado com restrições de capacidade e distância. Singh e Dhir (2014) apresentaram uma meta-heurística ACO para resolver o OVRP para ônibus escolares. Sedighpour et al. (2014) propuseram uma meta-heurística Ant Colony Optimization (ACO) hibrida e resolveram instâncias de testes do OVRP. Yao et al. (2014) aplicaram uma meta-heurística Ant Colony Optimization (ACO) para resolver OVRP com depósito central para o problema de roteamento de entregas de frutos do mar.

Mirhassani e Abolghasemi (2011) propuseram a meta-heurística Particle Swarm Optimization (PSO) para resolver o OVRP. Tarantilis et al. (2005) resolveram o OVRP aplicado ao planejamento de frota de veiculos alugados, usualmente utilizado por indústrias de distribuição de serviços. Para tal empregaram uma metaheurística de parâmetro único que explora uma lista de limiares para orientar de forma inteligente uma pesquisa local avançada. Pan e Fu (2009) apresentaram um algoritmo de seleção clonal (Clonal Selection Algorithm) para resolver o OVRP. Huang e Liu (2010) apresentaram um TS aperfeiçoada para resolver o OVRP, para isso utilizaram instâncias de problemas existentes na literatura. Wu e Tan (2009) propuseram uma meta-heurística PSO para resolver o OVRP aplicado ao roteamento de veículos na logística de grãos (GVRP).

Especificamente sobre o transporte de empregados por fretamento, destacam-se os autores a seguir: Raghavendra et al. (1992) desenvolveram um algoritmo heurístico para a resolução de uma variação do VRP associada à coleta e entrega de empregados de grandes organizações, que envolve fatores humanos e outras restrições específicas desse tipo de problema. Rosa (1996) propôs uma heurística específica para a solução do problema baseada na abordagem cluster first, route second aplicada ao transporte de empregados de uma grande empresa por uma frota de ônibus fretada. Salles (2013) utilizou o TransCAD para a coleta e distribuição física de empregados por uma frota de ônibus fretada limitando o trabalho a somente um bairro de uma cidade de médio porte. Perugia et al. (2011) apresentaram uma meta-heurística TS para modelar um serviço de transporte de empregados de uma empresa em uma área metropolitana. O modelo é multi-objetivo uma vez que visa um equilíbrio entre três critérios: eficiência, eficácia e equidade do transporte.

\section{MODELO MATEMÁTICO PROPOSTO}

O modelo matemático proposto nesse estudo teve como base o modelo proposto por Bektas e Elmastas (2007) ao qual foram introduzidos um limite inferiores e um superior em relação ao número de ônibus utilizados no transporte. Essa adaptação foi feita com o intuito de restringir o espaço de soluções, o que reduz substancialmente o tempo de execução do modelo. As reduções alcançadas são apresentadas e explicadas nas análises dos resultados do modelo.

Considerando que $q_{\text {tot }}$ é o número total de empregados a serem transportados e $Q$ a capacidade do ônibus de uma frota homogênea, define-se kmin e kmax como sendo, respectivamente, o limite mínimo e máximo de ônibus necessário para atender ao número total de empregados. O valor de kmin é calculado como sendo o número total de empregados dividido pela capacidade do ônibus, dado pela Equação (1). Tendo em vista que a cidade de Vitória não é geograficamente dispersa, e após a análise de várias rotas que eram executadas no dia a dia da empresa, definiu-se o valor de kmax como sendo $50 \%$ a mais do que o valor de kmin, conforme a Equação (2) onde perc $=1,5$.

$$
\begin{gathered}
k \min =\left[\frac{q_{t o t}}{Q}\right] \\
k \max =[1,5 \mathrm{kmin}]
\end{gathered}
$$

O modelo matemático proposto é apresentado a seguir em cinco partes: conjuntos, parâmetros, variáveis de decisão, função objetivo e restrições.

\section{Conjuntos:}

$I=$ Conjunto de pontos de ônibus;

$I_{0}=$ Conjunto auxiliar de pontos de ônibus incluindo a garagem;

$I_{1}=$ Conjunto auxiliar de pontos de ônibus incluindo a garagem virtual.

$N=$ Conjunto auxiliar de pontos de ônibus incluindo a garagem virtual.

\section{Parâmetros:}

$n$ = Número de pontos de ônibus;

$c d=$ Custo do quilômetro percorrido por ônibus;

$c f=$ Custo fixo de aluguel de um ônibus utilizado;

$v m=$ Velocidade média de percurso dos ônibus;

$q_{i}=$ Quantidade de passageiros em cada ponto de ônibus $i \in I$;

$T=$ Tempo máximo de viagem estabelecido;

$t_{i j}=$ Tempo de viagem entre o ponto $i \in I$ e o ponto $j \in I$.

\section{Variáveis de decisão:}

$u_{i}=$ Total acumulado de passageiros dentro do ônibus no ponto de ônibus $i$;

$v_{i}=$ Tempo de viagem total acumulado no ponto de ônibus $i$;

$k=$ Quantidade de ônibus utilizados no transporte;

$x_{i j}\left\{\begin{array}{l}1 \text { - Se o arco } \forall(i, j) \in I_{1} \text { é percorrido na solução; } \\ 0 \text { - Caso contrário. }\end{array}\right.$ 
Minimizar $c f k+\sum_{i \in N} \sum_{j \in N}\left(c d v m t_{i j} x_{i j}\right)$

Sujeito a:

$$
\begin{aligned}
& \sum_{i \in N} x_{0 i}=k \\
& \sum_{i \in I} x_{i(n+1)}=k \\
& \sum_{j \in I 1} x_{i j}=1 \\
& \sum_{i \in I 0} x_{i j}=1
\end{aligned}
$$

$$
\forall i \in I, i \neq j
$$

$$
\forall j \in I, i \neq j
$$

$k \min \leq k \leq k \max$

$$
u_{i}-u_{j}+Q x_{i j}+\left(Q-q_{i}-q_{j}\right) x_{j i} \leq Q-q_{j}
$$$$
\forall i, j \in I, i \neq j
$$

$$
u_{i} \geq q_{j}
$$$$
\forall i, j \in I, i \neq j
$$

$$
u_{i}-q_{i} x_{0 i}+Q x_{0 i} \leq Q
$$$$
\forall i \in I, i \neq j
$$

$$
v_{i}-v_{j}+\left(T-t_{i(n+1)}-t_{0 j}+t_{i j}\right) x_{i j}+\left(T-t_{i(n+1)}-t_{0 j}-t_{j i}\right) x_{j i} \leq T-t_{i(n+1)}-t_{0 j}
$$$$
\forall i, j \in I, i \neq j
$$

$$
v_{i}-t_{0 i} x_{0 i} \geq 0
$$$$
\forall i \in I, i \neq j
$$

$$
v_{i}-t_{0 i} x_{0 i}+T x_{0 i} \leq T
$$

$$
\forall i \in I, i \neq j
$$

Utilizando os parâmetros e variáveis de decisão descritos anteriormente, apresentam-se a seguir a função objetivo e as restrições do modelo proposto.

O objetivo do modelo é minimizar o valor do custo total do contrato do transporte. A Função Objetivo, dada pela Equação (3), representa a soma do custo total da distância percorrida mais o custo de aluguel dos ônibus. $\mathrm{O}$ custo total da distância percorrida é obtido pela somatória da multiplicação da distância, em quilômetros, entre os nós $i$ e $j$ pelo custo do quilômetro percorrido. Como o problema foi formulado considerando a variável tempo ao invés da distância, teve-se que realizar a conversão por meio da multiplicação do tempo dispendido ao atravessar o $\operatorname{arco}(i, j)$ pela velocidade média praticada pelos ônibus. O custo da utilização dos ônibus é obtido pela multiplicação do custo fixo contratado de um ônibus pelo número de ônibus encontrados pelo modelo.

As Restrições (4) e (5) estabelecem que $k$ ônibus partem do nó 0 (depósito) e chegam no nó $n+1$ (nó virtual). As Restrições (6) e (7) definem que cada nó intermediário pode ser visitado uma única vez. A Restrição (8) foi introduzida em função do limite inferior e superior propostos, kmin e kmax, visando limitar o intervalo possível para a variável $k$ e com isso reduzir o espaço de busca do modelo e, consequentemente, tornar menor o tempo computacional para encontrar a solução ótima. As Restrições (9), (10) e (11) são restrições de capacidade baseadas no trabalho de Miller et al. (1960) para o Capacitated Vehicle Routing Problem $(C V R P)$. Elas impõem que nenhum ônibus pode carregar mais que sua capacidade. As Restrições (9) evitam a formação de subrotas nos nós intermediários. As Restrições (12), (13) e (14) são restrições de tempo, as quais limitam o tempo total de viagem das rotas a um valor pré-determinado $T$. As Restrições (15) definem que a variável $x_{i j}$ assume somente valores 0 ou 1 .

\section{APRESENTAÇÃO E ANÁLISE DOS RESULTADOS}

Um ponto crítico para a realização deste trabalho foi o georreferenciamento dos pontos de ônibus, tendo em vista que a empresa estudada realiza o processo de roteirização de forma manual. Como os pontos utilizados no transporte de empregados são os mesmos utilizados pelo sistema público de transporte, utilizou-se a informação disponibilizada pela Prefeitura de Vitória sobre as coordenadas geográficas dos pontos de ônibus no Sistema Ponto Vitória (PMV, 2014).

O caso prático analisado nesse artigo contempla o transporte dos empregados dos turnos administrativo e operacional. Foram mapeados 89 pontos de ônibus da cidade de Vitória utilizados por 323 empregados de um turno administrativo e 94 pontos de ônibus utilizados para o transporte de 345 empregados de um turno operacional. As Figuras 2 (a) e 2 (b) mostram a distribuição desses pontos no mapa.

Uma vez conhecidas as coordenadas geográficas de dois pontos de ônibus distintos $(\phi$, pode-se calcular a distância esférica entre esses pontos (d) por meio da

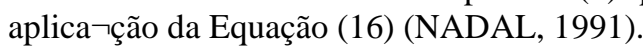




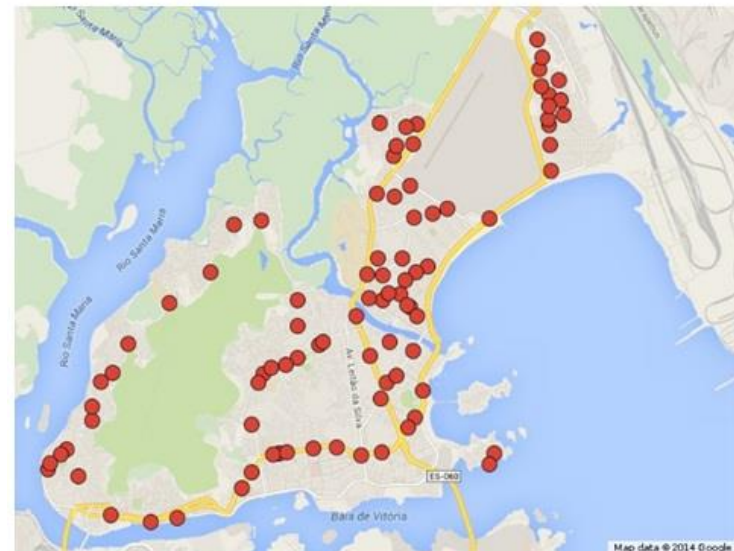

(a) Administrativo

Figura 2. Pontos de ônibus

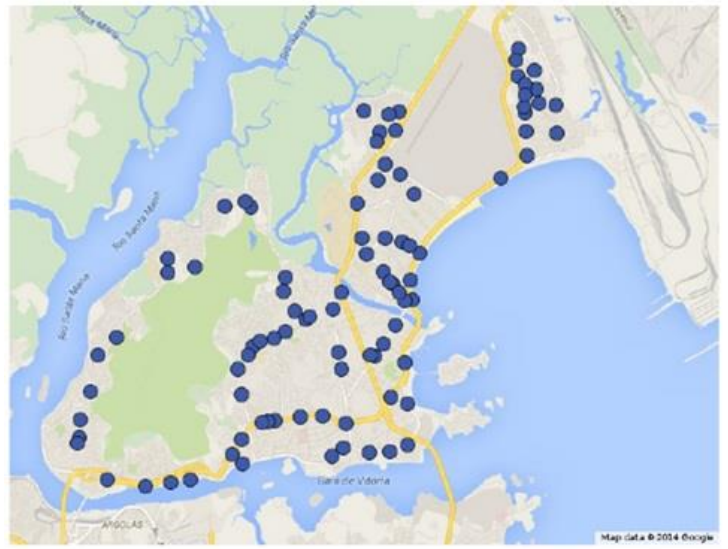

(b) Operacional

Fonte: Google Maps, adaptado pelos autores

A essa distância calculada foi aplicado um fator de correção, pois a distância esférica não considera a configuração da rede viária urbana. Segundo Novaes (1989), pode-se considerar esse fator de correção na ordem de $25 \%$.

$$
\cos d=\sin \varphi \cdot \sin \varphi^{\prime}+\cos \varphi \cdot \cos \varphi^{\prime} \cdot \cos \Delta \lambda
$$

Utilizou-se o solver CPLEX 12.6 para testar o modelo matemático proposto. Utilizou-se um computador com processador Intel Core i5 com 8GB de memória RAM para rodar o CPLEX e o tempo de execução máximo foi limitado em 7.200,0 segundos para cada instância.

Foram elaboradas 12 instâncias para analisar o comportamento e as influências dos parâmetros na solução do problema, além das instâncias 13 e 14, que se aproximam do cenário real praticado pela empresa para o transporte de empregados no município de Vitória nos turnos administrativo e operacional, respectivamente. As 12 instâncias de teste foram elaboradas com base em dados reais. Foram estabelecidas três quantidades de pontos de ônibus: 10, 20 e 30 pontos. Foram estabelecidos dois tipos de ônibus, 22 e 48 lugares. E, por fim, foram estabelecidos dois tempos limites de viagem, 1,0 e 2,0 horas. Então, para cada quantidade de ponto de ônibus, as outras duas características foram combinadas, gerando as 12 instâncias, Tabela 1. As doze primeiras instâncias de teste foram elaboradas com o intuito de compreender a influência dos parâmetros capacidade do ônibus e tempo máximo de viagem na solução do problema, além de serem úteis na verificação da viabilidade do modelo matemático. As instâncias propostas consideraram a possibilidade de a capacidade do ônibus ser de 22 ou 48 lugares, o que representa a utilização de micro-ônibus ou ônibus padrão para o transporte, e o tempo máximo de viagem variando entre 1,0 hora e 2,0 horas, sendo que o limite de tempo de viagem praticado atualmente pela empresa é de 1,5 horas. A partir da análise do resultado das doze instâncias é possível informar à empresa alvo deste estudo o cenário mais vantajoso em termos de custo, definindo qual veículo utilizar (ônibus ou micro-ônibus) e qual o tempo máximo de viagem adotar (maior ou menor que 1,5 horas). Foram levantados junto à empresa de ônibus que a velocidade média dos ônibus é de $24,72 \mathrm{Km} / \mathrm{h}$. Por questões de confi- dencialidade, a empresa analisada não permitiu a divulgação dos custos de aluguel de ônibus e do quilometro percorrido.

A Tabela 1 apresenta as instâncias de teste e as reais, sendo que: a coluna Instância contém um número sequencial que identifica unicamente cada instância dos testes e as demais colunas indicam as características da instância, a saber: o valor da coluna Nós corresponde a quantidade de pontos de ônibus considerados no problema; a coluna $\mathrm{Ca}$ pacidade do ônibus representa a capacidade máxima de ocupação do ônibus, ou seja, o número de assentos dos ônibus considerados naquela instância, sendo que 22 se refere à capacidade de um micro-ônibus e 48 de um ônibus padrão; a coluna Tempo máx. de viagem representa o tempo máximo permitido de permanência de um passageiro no ônibus e a coluna $\mathrm{N}^{\circ}$ total de passageiros representa a demanda de transporte, ou seja, o número total de passageiros que devem ser atendidos naquela instância.

Tabela 1. Instâncias analisadas pelo modelo

\begin{tabular}{ccccc}
\hline Instância & Nós & $\begin{array}{c}\text { Capacidade } \\
\text { do ônibus } \\
\text { (um) }\end{array}$ & $\begin{array}{c}\text { Tempo } \\
\text { máx. de } \\
\text { viagem } \\
\text { (h) }\end{array}$ & $\begin{array}{c}\text { Número } \\
\text { total de } \\
\text { passageiros } \\
\text { (um) }\end{array}$ \\
\hline 1 & 10 & 22 & 1,0 & 28 \\
2 & 10 & 22 & 2,0 & 28 \\
3 & 10 & 48 & 1,0 & 28 \\
4 & 10 & 48 & 2,0 & 28 \\
5 & 20 & 22 & 1,0 & 46 \\
6 & 20 & 22 & 2,0 & 46 \\
7 & 20 & 48 & 1,0 & 46 \\
8 & 20 & 48 & 2,0 & 46 \\
9 & 30 & 22 & 1,0 & 86 \\
10 & 30 & 22 & 2,0 & 86 \\
11 & 30 & 48 & 1,0 & 86 \\
12 & 30 & 48 & 2,0 & 86 \\
13 & 89 & 48 & 1,5 & 323 \\
14 & 94 & 48 & 1,5 & 345 \\
\hline
\end{tabular}

Os resultados obtidos após a execução do modelo no CPLEX para as doze instâncias de teste e as duas instâncias que representam o Caso Real estão apresentados na Tabela 2 onde a coluna Instância representa as instâncias analisadas. As colunas FO, UB, LB apresentam, respectivamente, a Função Objetivo (FO) do modelo, que corresponde ao custo total do transporte, o Upper Bound (UB) e o Lower Bound (LB), que são os limites superior e inferior no caso de não obtenção de solução ótima. As colunas TE e GAP representam o tempo de execução do 
Tabela 2. Resultados obtidos pelo CPLEX

\begin{tabular}{|c|c|c|c|c|c|c|c|c|c|}
\hline Instância & FO (R\$) & UB (R\$) & $\mathbf{L B}(\mathbf{R} \$)$ & TE (s) & GAP $(\%)$ & $\begin{array}{c}\mathbf{N}^{\circ} \text { de } \\
\text { ônibus } \\
\text { utilizados } \\
\text { (um) }\end{array}$ & $\begin{array}{c}\text { Taxa } \\
\text { média de } \\
\text { ocupação } \\
(\%)\end{array}$ & $\begin{array}{l}\text { Tempo } \\
\text { médio de } \\
\text { viagem } \\
\text { (h) }\end{array}$ & $\begin{array}{c}\text { Distância } \\
\text { média } \\
\text { percorrida } \\
(\mathbf{k m})\end{array}$ \\
\hline 1 & 46028,6 & - & - & 0,02 & 0 & 2 & 63,64 & 0,47 & 11,63 \\
\hline 2 & 46028,6 & - & - & 0,01 & 0 & 2 & 63,64 & 0,47 & 11,63 \\
\hline 3 & 19026,09 & - & - & 2,17 & 0 & 1 & 58,33 & 0,70 & 17,39 \\
\hline 4 & 19026,09 & - & - & 2,62 & 0 & 1 & 58,33 & 0,70 & 17,39 \\
\hline 5 & 69047,92 & - & - & 0,44 & 0 & 3 & 69,7 & 0,53 & 12,99 \\
\hline 6 & 69049,97 & - & - & 0,36 & 0 & 3 & 69,7 & 0,55 & 13,54 \\
\hline 7 & 19034,47 & - & - & 0,16 & 0 & 1 & 95,83 & 0,93 & 22,98 \\
\hline 8 & 19034,47 & - & - & 0,11 & 0 & 1 & 95,83 & 0,93 & 22,98 \\
\hline 9 & 92065,01 & - & - & 384,36 & 0 & 4 & 97,73 & 0,53 & 13,21 \\
\hline 10 & 92064,08 & - & - & 643,33 & 0 & 4 & 97,73 & 0,53 & 13,02 \\
\hline 11 & 38052,73 & - & - & 6,41 & 0 & 2 & 89,58 & 0,71 & 17,58 \\
\hline 12 & 38052,97 & - & - & 4,79 & 0 & 2 & 89,58 & 0,71 & 17,66 \\
\hline 13 & - & 133090,5 & 133090,5 & 7200,00 & 0,05 & 7 & 96,13 & 0,59 & 14,66 \\
\hline 14 & - & 152137,7 & 152086,5 & 7200,00 & 0,03 & 8 & 89,84 & 0,46 & 11,48 \\
\hline
\end{tabular}

CPLEX e o GAP respectivamente, sendo que o GAP é calculado como sendo GAP $=100 \times(\mathrm{UB}-\mathrm{LB}) / \mathrm{UB}$.

Nas colunas $\mathrm{N}^{\mathrm{o}}$ de ônibus utilizados, Taxa média de ocupação, Tempo médio de viagem e Distância média percorrida representam, respectivamente, o número de ônibus utilizados no transporte, a taxa média de ocupação dos ônibus, o tempo médio de viagem e a distância média percorrida pelos ônibus.

Analisando os resultados da Tabela 2, percebe-se que, na maioria das instâncias, foi possível chegar a uma solução ótima, já que o GAP apresentado foi $0,0 \%$ para as Instâncias de 1 a 12. No caso das Instâncias 13 e 14, obtevese, respectivamente, GAP de $0,05 \%$ e $0,03 \%$ após um tempo de execução de 2,0 horas, que foi o limite estabelecido para a execução do CPLEX. Embora não se tenha obtido uma solução ótima para as instâncias que representam o Caso Real, percebeu-se que, durante a sua execução, o CPLEX provavelmente já havia encontrado uma solução próxima da ótima, uma vez que, além do GAP ser um valor muito pequeno, o CPLEX permaneceu com o mesmo GAP por um longo tempo de execução, além do que o UB não se alterou e o LB foi aumentando muito lentamente.

Ao executar as Instâncias 13 e 14 sem aplicar o limite inferior e o limite superior ao número de ônibus proposto neste artigo, o CPLEX apresentou GAP de 99,94\% e

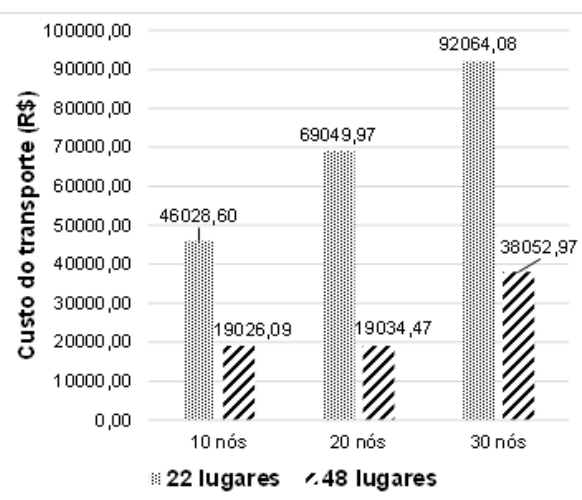

(a) Influência da capacidade do ônibus no custo total do transporte os GAP caíram para $0,05 \%$ e $0,03 \%$ respectivamente. Vale ressaltar que, para as Instâncias 13 e 14, como não foi obtida a solução ótima, utilizou-se o valor do Upper Bound a fim de se estimar o tempo médio de viagem e a distância média percorrida pelos ônibus na situação mais desvantajosa, pois a FO pode estar em um valor menor que o UB e superior ao LB.

Ao analisar somente as Instâncias 1 a 12, percebeuse que a utilização de ônibus padrão é mais vantajosa em termos de custo de transporte que a de micro-ônibus. Isso ocorre, primeiramente, porque o custo do aluguel do microônibus é mais elevado que o do padrão.

O gráfico da Figura 3 (a) mostra que a utilização de ônibus padrão apresenta menores custos de transporte que a de micro-ônibus nas instâncias de teste com 10, 20 e 30 pontos de ônibus. O parâmetro Tempo Máximo de Viagem (TMV) não apresenta influência no custo total de transporte (FO). Isso foi percebido ao comparar instâncias que diferem somente em relação ao tempo de viagem, conforme apresentado no gráfico da Figura 3 (b).

Analisando o gráfico ]da Figura 3 (b), percebeu-se que o custo de transporte nas instâncias de teste com 10, 20 e 30 pontos de ônibus é idêntico quando se varia o parâmetro TMV entre 1,0 hora e 2,0 horas de viagem. Dessa forma, como é vantagem para o empregado um menor $\mathrm{TMV}$, pois este permanece menos tempo no interior do ônibus, a empresa pode estudar as instâncias em que seja

Figura 3. Legenda 3

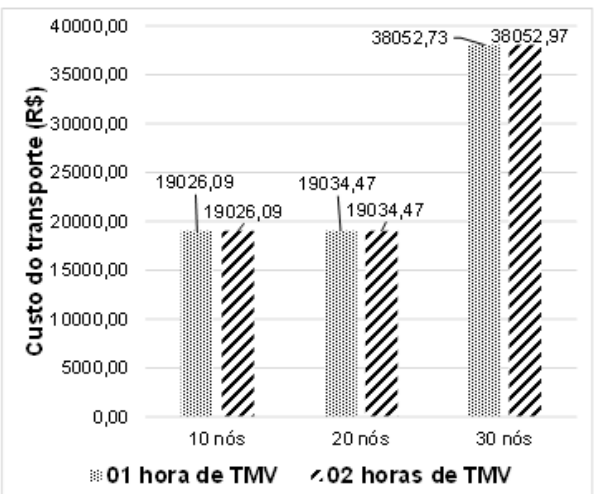

(b) Influência do Tempo Máximo de Viagem no custo total do transporte

99,95\% respectivamente. Com a introdução destes limites, 
SCÁRDUA, R.F.; ROSA, R.A.; SABINO, J.A.; VITORUGO, L.R.

Tabela 3. Comparação do CPLEX versus o Caso Real

\begin{tabular}{lcccccc}
\hline \multirow{2}{*}{ Índices de Comparação } & \multicolumn{2}{c}{ Caso Real } & \multicolumn{2}{c}{ CPLEX } & \multicolumn{2}{c}{$\begin{array}{c}\text { Redução CPLEX - } \\
\text { Caso Real (\%) }\end{array}$} \\
\cline { 2 - 7 } & Adm & Ope & Adm & Ope & Adm & Ope \\
\hline Ônibus utilizados (um) & 10 & 9 & 7 & 8 & 30 & 11 \\
\hline Taxa de ocupação (\%) & 48,86 & 59,48 & 96,13 & 89,84 & -97 & -51 \\
\hline Tempo de viagem (h) & 0,38 & 0,38 & 0,59 & 0,46 & -55 & -21 \\
\hline Distância média percorrida $(\mathrm{km})$ & 9,46 & 9,74 & 14,66 & 11,48 & -55 & -21 \\
\hline Distância percorrida por dia $(\mathrm{km})$ & 94,60 & 85,23 & 102,63 & 91,82 & -8 & -8 \\
\hline Distância total por mês $(\mathrm{km})$ & $4.162,40$ & $3.750,12$ & $4.515,72$ & $4.040,08$ & -8 & -8 \\
\hline
\end{tabular}

Tabela 4. Ganho do CPLEX em relação ao Caso Real

\begin{tabular}{lcccccccc} 
& \multicolumn{3}{c}{$\begin{array}{c}\mathbf{N}^{\circ} \text { de veículos } \\
\text { utilizados }(\text { um) }\end{array}$} & \multicolumn{2}{c}{$\begin{array}{c}\text { Distância total } \\
\text { percorrida }(\mathbf{k m})\end{array}$} & \multicolumn{2}{c}{ Custo (R\$) } & \multicolumn{2}{c}{$\begin{array}{c}\text { Ganho } \\
\text { (CPLEX x REAL) }\end{array}$} \\
\cline { 2 - 10 } & Caso Real & CEPLEX & Caso Real & CEPLEX & Caso Real & CEPLEX & R\$ & \% \\
\hline Turno ADM & 10 & 7 & $4.162,40$ & $4.515,72$ & $196.243,60$ & $139.773,58$ & $56.470,02$ & 28,78 \\
\hline Turno OPE & 9 & 8 & $3.750,12$ & $4.040,08$ & $176.625,18$ & $158.060,12$ & $18.565,06$ & 10,51 \\
\hline Total & 19 & 15 & $7.912,52$ & $8.555,80$ & $372.868,78$ & $297.833,70$ & $75.035,08$ & 20,12 \\
\hline
\end{tabular}

reduzido o tempo de viagem dos empregados, já que essa mudança não afetará o custo total de transporte.

O TMV só exerce influência no tempo de execução do CPLEX. De uma forma geral, observou-se que instâncias com a restrição de limite de tempo de viagem de 2,0 horas apresentaram solução ótima relativamente mais rápido que as instâncias com o limite de 1,0 hora de viagem.

Dados reais referentes ao praticado pela empresa estudada foram coletados com o intuito de realizar uma comparação entre os resultados do CPLEX e o Caso Real. Os dados reais coletados foram multiplicados por uma constante para preservar a confidencialidade dos dados da empresa. A Tabela 3 evidencia esta comparação em termos de número de ônibus utilizados no transporte, taxa de ocupação média dos ônibus, tempo médio de viagem e distância média percorrida. Observou-se que o CPLEX obteve ganhos nos dois casos, administrativo (Adm) e operacional (Ope), em relação ao Caso Real. A distância total por mês foi calculada tomando como base um mês de 22 dias úteis e considerando que o ônibus percorre a rota duas vezes ao dia, uma para trazer os empregados à empresa e outra para levar os empregados para casa. Ambas as rotas são iguais.

Apesar da solução do CPLEX apresentar um acréscimo na distância total percorrida pelos ônibus nos dois turnos de trabalho, de $7.912,52 \mathrm{~km}$ para $8.555,80 \mathrm{~km}$, o CPLEX obteve uma redução total do custo em função da redução da soma dos ônibus utilizados nos dois turnos de trabalho, de 19 no Caso Real para 15 no resultado do CPLEX. Esta diminuição foi possível por meio do aumento da taxa de ocupação dos ônibus conforme pode ser constatado na Tabela 3.

Para estimar o custo total de transporte, tanto na solução do CPLEX quanto no Caso Real, foi realizada uma consulta às empresas de transportes onde obteve-se o custo do frete médio do ônibus padrão de $\mathrm{R} \$ 19.000,00$ e o custo médio do quilômetro rodado de $\mathrm{R} \$ 1,50$. Os resultados da comparação podem ser vistos na Tabela 4.

Como pode ser visto na Tabela 4, a solução apresentada produz um ganho mensal de $\mathrm{R} \$ 75.035,08$ e um ganho anual estimado de $\mathrm{R} \$ 900.000,00$ no transporte dos turnos operacional e administrativo em Vitória. Este ganho representa uma redução de aproximadamente $20 \%$ do custo total de transporte atual. Assim, pode-se notar que a solução proposta é capaz de produzir ganhos financeiros interessantes para a empresa. Ao reduzir o custo da logística de transporte de empregados, a empresa pode reverter estes valores em investimentos que melhorem a qualidade de vida de seus empregados, tais como a aquisição de ônibus mais confortáveis dentre outros.

\section{CONCLUSÃO}

O presente trabalho teve como objetivo aplicar um modelo matemático baseado no Open Vehicle Routing Problem para o planejamento da logística de transporte de empregados por uma frota de ônibus fretada. Foram introduzidos em um modelo da literatura dois limitantes para o número de ônibus, um inferior e um superior, visando reduzir o tempo computacional para encontrar a solução ótima. Estes limitantes se mostraram muito importantes, pois contribuíram para convergir mais rapidamente para uma solução ótima do problema e/ou reduziram substancialmente o GAP encontrado pelo CPLEX a valores aceitáveis para uma solução aplicável na prática.

O modelo adaptado foi aplicado ao transporte dos empregados dos turnos administrativo e operacional, em seus trajetos residência-trabalho-residência no município de Vitória. Observou-se que a utilização do modelo no auxílio ao planejamento do transporte, pode proporcionar um ganho financeiro mensal de $\mathrm{R} \$ 75.035,08$ e um ganho anual da ordem de R $\$ 900.000,00$. Este ganho corresponde a uma redução aproximada de $20 \%$ no custo de transporte da empresa.

Apesar dos resultados do CPLEX apresentarem um acréscimo na distância total percorrida pelos ônibus, de $7.912,52 \mathrm{~km}$ para $8.555,80 \mathrm{~km}$, foi obtido em contrapartida um ganho significativo devido à redução do número de ônibus utilizados no transporte, de 19 para 15, considerando os dois turnos de trabalho. Ao reduzir o custo da logística de transporte de empregados, a empresa pode reverter estes valores em investimentos que melhorem a qualidade de vida de seus empregados, tais como a aquisição de ônibus mais confortáveis dentre outros.

Outra contribuição do modelo à empresa alvo deste estudo foi provê-la de uma sistematização da roteirização de veículos mais confiável e flexível. Como atualmente o trabalho de concepção e atualização de rotas é realizado de 
forma empírica e por poucos empregados, o modelo proposto eliminaria a necessidade desse trabalho, tornando essa atividade um processo automático. $\mathrm{O}$ modelo se mostrou, portanto, uma ferramenta importante tanto na redução de custos logísticos quanto na melhoria do processo de concepção e gestão das rotas. O modelo se mostra igualmente aplicável em problemas de mesma natureza, como transporte escolar, transporte para eventos e turismo, entre outros. Porém, o modelo matemático possui uma limitação quando aplicado a problemas de grande porte devido ao tempo computacional ser muito elevado.

Por fim, sugere-se para trabalhos futuros o desenvolvimento de uma meta-heurística visando a solução de problemas de maior porte.

\section{AGRADECIMENTOS}

Os autores agradecem à FAPES (458/2013) e ao CNPq (477357/2013-0, 128033/2014-2) pelo apoio financeiro.

\section{REFERÊNCIAS}

Bektaş, T.; Elmastaş, Seda. Solving school bus routing problems through integer programming. Journal of the Operational Research Society, v. 58, n. 12, p. 1599-1604, 2007. DOI: $10.1057 /$ palgrave.jors.2602305

Bodin, L.; Golden, B.; Assad, A. routing and scheduling of vehicles and crews-the state of the art. 1981.

Brandão, José. A tabu search algorithm for the open vehicle routing problem. European Journal of Operational Research, v. 157 , n. 3, p. 552-564, 2004. DOI: 10.1016/S0377-

2217(03)00238-8

De Jaegere, Nathalie; Defraeye, Mieke; Van Nieuwenhuyse, Inneke. The vehicle routing problem: state of the art classification and review. 2014.

Fu, Z.; Eglese, R.; Li, L. Y. O. A new tabu search heuristic for the open vehicle routing problem. Journal of the Operational Research Society, v. 57, n. 8, p. 1018-1018, 2006.

DOI: $10.1057 /$ palgrave.jors.2602181

HUANG, Fuhua; LIU, Changshi. An improved tabu search for open vehicle routing problem. In: Management and Service Science (MASS), 2010 International Conference on. IEEE, 2010. p. 1-4. DOI: 10.1109/ICMSS.2010.5576368

Letchford, Adam N.; Lysgaard, Jens; Eglese, Richard W. A branch-and-cut algorithm for the capacitated open vehicle routing problem. Journal of the Operational Research Society, v. 58, n. 12, p. $1642-1651,2007$. DOI: $10.1057 /$ palgrave.jors. 2602345

LI, Feiyue; GOLDEN, Bruce; WASIL, Edward. The open vehicle routing problem: Algorithms, large-scale test problems, and computational results. Computers \& operations research, v. 34 , n. 10 , p. 2918-2930, 2007. DOI: 10.1016/j.cor.2005.11.018

Li, Xiangyong; Tian, Peng. An ant colony system for the open vehicle routing problem. In: International Workshop on Ant Colony Optimization and Swarm Intelligence. Springer Berlin Heidelberg, 2006. p. 356-363. DOI: 10.1007/11839088_33

Miller, Clair E.; Tucker, Albert W.; Zemlin, Richard A. Integer programming formulation of traveling salesman problems.
Journal of the ACM (JACM), v. 7, n. 4, p. 326-329, 1960. DOI: $10.1145 / 321043.321046$

Mirhassani, S. A.; Abolghasemi, N. A particle swarm optimization algorithm for open vehicle routing problem. Expert Systems with Applications, v. 38, n. 9, p. 11547-11551, 2011. DOI: 10.1016/j.eswa.2011.03.032

Nadal, C. A. Introdução à Trigonometria Esférica - Aplicações na Astronomia e na Cartografia. Curitiba. 1991.

Novaes, Antonio. Logistica e Gerenciamento da Cadeia de Distribuição-4a Edição. Elsevier Brasil, 2004.

Pan, Lijun; Fu, Z. A Clone Selection Algorithm for the Open Vehicle Routing Problem. In: 2009 Third International Conference on Genetic and Evolutionary Computing. 2009. DOI: 10.1109/WGEC.2009.174

Perugia, Alessandro et al. Designing a home-to-work bus service in a metropolitan area. Transportation Research Part B: Methodological, v. 45, n. 10, p. 1710-1726, 2011. DOI: $10.1016 /$ j.trb.2011.05.025

Pinto, V. P. A inserção do transporte por fretamento na matriz da mobilidade urbana. Série Cadernos Técnicos, Campinas, v. 9, p. 12-25, 2012.

PMV - Sistema Ponto Vitória. Disponível em: <www.vitoria.es.gov.br/pontovitoria〉. Acesso em: 04 Out. 2014.

Raghavendra, Anirudh et al. A practical heuristic for a large scale vehicle routing problem. European journal of operational research, v. 57 , n. 1, p. 32-38, 1992. DOI: 10.1016/03772217(92)90303-Q

Rosa, R.A. Roteirização do transporte diário de empregados por uma frota de ônibus fretada. 1996. 119 p. Dissertação (Mestrado em Informática) - Programa de Pós-Graduação em Informática, Universidade Federal do Espírito Santo, Vitória, 1996.

Salari, Majid; TOTH, Paolo; TRAMONTANI, Andrea. An ILP improvement procedure for the open vehicle routing problem. Computers \& Operations Research, v. 37, n. 12, p. 2106-2120, 2010. DOI: 10.1016/j.cor.2010.02.010

Salles, R. S. Estudo de roteirização de veículos com apoio de um sistema de informações geográficas: uma contribuição para o transporte urbano de empregados por uma frota de ônibus fretada. 2013. 159 p. Dissertação (Mestrado em Engenharia Civil) - Programa de Pós-Graduação em Engenharia Civil, Universidade Federal do Espírito Santo, Vitória, 2013.

Sariklis, Dimitrios; Powell, Susan. A heuristic method for the open vehicle routing problem. Journal of the Operational Research Society, v. 51, n. 5, p. 564-573, 2000. DOI: $10.1057 /$ palgrave.jors. 2600924

Sedighpour, Mohammad et al. Solving the open vehicle routing problem by a hybrid ant colony optimization. Kuwait Journal of Science, v. 41, n. 3, 2014.

Singh, Er Gurpreet; Dhir, Vijay. Open Vehicle Routing Problem by Ant Colony Optimization. International Journal of Advanced Computer Science \& Applications, v. 1, n. 5, p. 63-68. DOI: 10.1.1.682.3142

Tarantilis, C. D.; Kiranoudis, C. T. Distribution of fresh meat. Journal of Food Engineering, v. 51, n. 1, p. 85-91, 2002. DOI: $10.1016 /$ S0260-8774(01)00040-1 
Tarantilis, C. D. et al. Solving the open vehicle routeing problem via a single parameter metaheuristic algorithm. Journal of the Operational Research Society, v. 56, n. 5, p. 588-596, 2005. DOI: http://dx.doi.org/10.1057/palgrave.jors.2601848

Vasconcellos, E. A. Os serviços de transporte de passageiros por fretamento. Série Cadernos Técnicos, Campinas, v. 9, p. 26-45, 2012.

Wu, Jianjun; Tan, Yubo. A particle swarm optimization algorithm for grain logistics vehicle routing problem. In: 2009 ISECS International Colloquium on Computing,

Communication, Control, and Management. IEEE, 2009. p. 364 367. DOI: 10.1109/CCCM.2009.5267915

Yao, Baozhen et al. Improved ant colony optimization for seafood product delivery routing problem. PROMET-

Traffic\&Transportation, v. 26, n. 1, p. 1-10, 2014. DOI: http://hrcak.srce.hr/124151

Yu, Shiwei; Ding, Chang; Zhu, Kejun. A hybrid GA-TS algorithm for open vehicle routing optimization of coal mines material. Expert Systems with Applications, v. 38, n. 8, p. 1056810573, 2011. DOI: 10.1016/j.eswa.2011.02.108

Zachariadis, Emmanouil E.; Kiranoudis, Chris T. An open vehicle routing problem metaheuristic for examining wide solution neighborhoods. Computers \& Operations Research, v. 37, n. 4, p. 712-723, 2010. DOI: 10.1016/j.cor.2009.06.021 